\title{
Verification of Cefmetazole and Cefpodoxime Proxetil Contamination to Other Pharmaceuticals by Liquid Chromatography-Tandem Mass Spectrometry
}

\author{
Naoto FukUtsu, ${ }^{*}, a$ Yuich SAKamaki, ${ }^{b}$ Takao Kanasaki, ${ }^{a}$ Koichi Saito, ${ }^{c}$ and Hiroyuki NaKazawa ${ }^{c}$ \\ ${ }^{a}$ Analytical and Quality Evaluation Research Laboratories, Sankyo Co., Ltd.; ${ }^{b}$ Quality Control Section, Hiratsuka Plant, \\ Sankyo Co., Ltd.; 1-12-1 Shinomiya, Hiratsuka-shi, Kanagawa 254-0014, Japan: and ' Department of Analytical \\ Chemistry, Faculty of Pharmaceutical Sciences, Hoshi University; 2-4-41 Ebara, Shinagawa-ku, Tokyo, 142-8501, Japan. \\ Received June 2006; accepted July 26, 2006
}

Cross-contamination is a critical issue for pharmaceutical manufacturing, especially for $\beta$-lactam antibiotics. Thus, an analytical method for the simultaneous determination of $\beta$-lactam antibiotics cefmetazole (CMZ) and cefpodoxime proxetil (CPDXPR) contaminants in non- $\beta$-lactam pharmaceuticals was developed using highperformance liquid chromatography-tandem mass spectrometry. The developed method was found to be sensitive at the detection limit of $\mathbf{0 . 0 0 2} \mathbf{~ p p m}$ for both compounds. Mean recoveries of CMZ and CPDXPR from olmesartan medoxomil (OLM) tablets were 96.7 to $102.2 \%$ and 88.9 to $94.2 \%$, respectively. The developed method was successfully applied for the verification of CMZ and CPDXPR contamination to actually manufactured OLM tablets.

Key words $\beta$-lactam antibiotic; contamination; liquid chromatography-tandem mass spectrometry

Pharmaceuticals contaminated with highly sensitizing or highly potent pharmaceuticals may cause unexpected adverse reactions. A serious potential health risk to patients by administering pharmaceuticals contaminated with diethylstilbestrol was found in the $\left.1960 \mathrm{~s} .{ }^{1,2}\right)$ Since then, regulatory agencies have issued guidelines to control contamination of pharmaceuticals.

Cephamycin antibiotic, cefmetazol (CMZ) and cephem antibiotic, cefpodoxime proxetil (CPDXPR), are semi-synthetic $\beta$-lactam antibiotics. CMZ was developed as a 7-alphamethoxy cephalosporin used for intravenous injection. CPDXPR was developed as an esterified cephalosporin at the 4-carboxyl group and consists of a mixture of two diastereomers arising from the stereogenic center at the carboxyl ester moiety used for oral administration. They have a broad spectrum against gram negative and positive bacteria, and are widely used for infectious diseases. However, as typified by penicillins and cephalosporins, $\beta$-lactam antibiotics are well known to cause serious anaphylaxis in rare cases, ${ }^{3-6)}$ thus pharmaceuticals contaminated with CMZ or CPDXPR may induce anaphylactic shock in some patients.

In order to prevent contamination of pharmaceuticals at a manufacturing facility, an adequate cleaning procedure for the manufacturing equipment and a validated analytical method for the monitoring of pharmaceutical residues are required. $^{7-9)}$ As analytical methods for the determination of pharmaceutical residues at manufacturing facilities, the HPLC-UV method ${ }^{10-13)}$ and total organic carbon (TOC) method $^{14)}$ have generally been used. However, in spite of universal application of these methods for detection of pharmaceutical residues, their application to monitoring of trace amounts of residues or contaminants of highly potent or sensitizing pharmaceuticals is limited due to their lack of selectivity and sensitivity. Thus, a sensitive luminol chemiluminescence-flow injection analysis method and a high-performance liquid chromatography-tandem mass spectrometry (LCMS/MS) method were developed for $\beta$-lactam antibiotics CMZ and CPDXPR and applied to verify their contamination to the manufacturing equipment and environment at a facility. ${ }^{15,16)}$ However, the contamination of CMZ and CPDXPR to other pharmaceutical products had not been verified with actually manufactured pharmaceutical products, nor had a method for the determination of CMZ or CPDXPR contaminants in other pharmaceuticals been developed.

In the present study, therefore, a simultaneously sensitive and selective LC-MS/MS method for the determination of CMZ and CPDXPR contaminants in other non- $\beta$-lactam pharmaceutical products was investigated to verify their contamination. Since the drug product of the anti-hypertensive agent olmesartan medoxomil (OLM) is manufactured at same manufacturing site as CMZ and CPDXPR, OLM tablets were used in this study as non- $\beta$-lactam pharmaceutical products.

\section{Experimental}

Materials and Methods $\beta$-Lactam antibiotics, cephamycin antibiotic CMZ and cephem antibiotic CPDXPR, and an anti-hypertensive agent, OLM, synthesized by Sankyo Co., Ltd. (Tokyo, Japan), were used. The chemical structures of these compounds are shown in Fig. 1. OLM 5, 20 and $40 \mathrm{mg}$ tablets manufactured at plants in Sankyo Co., Ltd. (Tokyo, Japan) and Sankyo Pharma GmbH (Munich, Germany) were used.

Acetonitrile of high-performance liquid chromatography grade was purchased from Merck Ltd. (Frankfurt, Germany). Formic acid of guaranteed grade and water of high performance liquid chromatography grade were purchased from Wako Pure Chemical Industries, Ltd. (Osaka, Japan).

HPLC An Agilent 1100 HPLC system (Agilent Technologies, Palo Alto, CA, U.S.A.), consisting of an online degasser (G1379A), a binary pump (G1312A), an autosampler (G1329A), a column compartment (G1316A) and a diode array detector (G1315B), with a switching valve compartment was used. The separation was performed using as the analytical column for reversed-phase chromatography Develosil C30-UG-3, $3 \mu \mathrm{m}$ in particle size, $2.0 \mathrm{~mm}$ in inside diameter and $150 \mathrm{~mm}$ in length (Nomura Chemical Co., Ltd., Aichi, Japan). A mixture of water, acetonitrile and formic acid $(550: 450: 1, \mathrm{v} / \mathrm{v} / \mathrm{v})$ was used as the mobile phase. Aliquots of $10 \mu 1$ of the analytical solutions were injected into the system, and separation was achieved with the analytical column kept at a constant temperature of $40^{\circ} \mathrm{C}$ with flow rate of $0.2 \mathrm{ml} / \mathrm{min}$

Mass Spectrometry A TSQ 7000 triple quadrupole mass spectrometer (Thermo Electron, Waltham, MA, U.S.A.) equipped with an electorospray ionisation source was operated in the positive ionization mode. The capillary voltage was set at $4.5 \mathrm{kV}$. Nitrogen was provided for both the sheath gas at a 
pressure of $70 \mathrm{psi}$ and the auxiliary gas at a flow rate of 40 arbitrary units. The heated capillary was maintained at a temperature of $200^{\circ} \mathrm{C}$. Argon was used as the collision gas, and the magnetic field of the gas cell was adjusted to $2 \mathrm{mT}$ for the MS/MS conditions.

Preparation of Standard and Sample Solution for Actual Sample Twenty OLM $5 \mathrm{mg}$ tablets, ten OLM $20 \mathrm{mg}$ tablets and five OLM $40 \mathrm{mg}$ tablets were each transferred into a $100-\mathrm{ml}$ volumetric flask, respectively. To each tablet, $80 \mathrm{ml}$ of a mixture of water and acetonitrile $(11: 9, \mathrm{v} / \mathrm{v})$ was added, and the mixture was sonicated with occasional shaking until the tablets disintegrated completely. Then a mixture of water and acetonitrile $(11: 9, \mathrm{v} / \mathrm{v})$ was added to volume. This solution was centrifuged at $3000 \mathrm{rpm}$ for $5 \mathrm{~min}$, and then the supernatant was filtrated through a polytetrafluoroethylene filter of $0.45 \mu \mathrm{m}$ in pore size. The resultant solution was used as the sample solution.

Separately, CMZ and CPDXPR of $65 \mathrm{mg}$ each were weighed accurately and transferred together into a 100-ml volumetric flask. Then, CMZ and CPDXPR were dissolved with a mixture of water and acetonitrile (11:9, $\mathrm{v} / \mathrm{v})$ to volume. The solution was further diluted with a mixture of water and acetonitrile $(11: 9, \mathrm{v} / \mathrm{v})$ to a concentration of $0.65 \mathrm{ng} / \mathrm{ml}$.

Preparation of Solutions for Validation Study In order to verify specificity, non-contaminated OLM tablets were subjected to evaluation. The sample solutions of the tablets were prepared in accordance with the procedure for actual OLM tablets. For linearity, CMZ and CPDXPR of $65 \mathrm{mg}$ each were dissolved and diluted to $100 \mathrm{ml}$ with acetonitrile. The solution was diluted with a mixture of water and acetonitrile $(11: 9, \mathrm{v} / \mathrm{v})$ to obtain various concentrations in the range of 0.325 to $3.25 \mathrm{ng} / \mathrm{ml}$. These solutions were subjected to demonstrate linearity of the method. Accuracy and precision testing were demonstrated by determination of known amounts of CMZ and CPDXPR in OLM tablets. The solutions containing 3.25, 6.50 and $13.00 \mathrm{ng}$ of $\mathrm{CMZ}$ and CPDXPR were added to non-contaminated 5, 20 and $40 \mathrm{mg}$ OLM tablets, respectively. Then, sample solutions were prepared according to the procedure for actual OLM tablets.

\section{Results and Discussion}

Optimization of MS/MS Conditions A selected reaction monitoring (SRM) method was chosen and investigated to achieve the necessary selectivity and sensitivity of the analytical method. SRM conditions were determined based on the data of MS/MS spectra as shown in Fig. 2. A protonated molecule at $\mathrm{m} / \mathrm{z} 472$ as a precursor ion and a fragment at $\mathrm{m} / \mathrm{z}$ 356 as a monitor ion were selected for $\mathrm{CMZ}$ determination with collision energy of $11 \mathrm{eV}$. For CPDXPR, a protonated molecule at $\mathrm{m} / z 558$ as a precursor ion and a fragment at $\mathrm{m} / \mathrm{z}$ 410 as a monitor ion were selected with collision energy of $17 \mathrm{eV}$. Under these conditions, however, OLM interfered with detection of CPDXPR in the sample solution. This was because the ion masses of the precursor and monitor ions between OLM and CPDXPR differed by only one mass unit, and the resolution of the mass spectrometer was not sufficient to distinguish their ions. The $\mathrm{m} / z$ value of the OLM protonated molecule was 559, and a small fragment ion at $\mathrm{m} / \mathrm{z} 411$ was formed from the molecule ion as shown in Fig. 2. Concurrent with this, as shown in the chromatogram monitored by UV in Fig. 3, a large OLM peak was eluted just before the peak of CPDXPR, and thus the small fragment ion at $m / z 411$ in OLM had a significant influence on the detection of CPDXPR. From these reasons, the CPDXPR was not detected in the sample solution.

Then, instead of the fragment ion at $m / z 410$, another fragment ion at $m / z$ 241, which was not observed in OLM, was

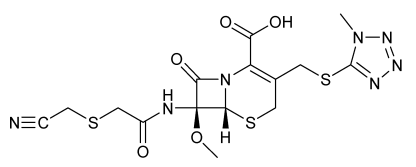

Cefmetazole (CMZ)

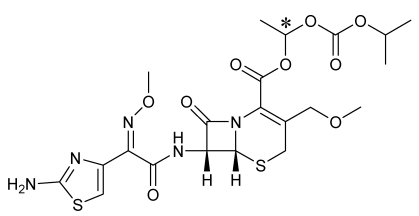

Cefpodoxime proxetil (CPDXPR)

*A mixture of $(R)$ and $(S)$ isomers

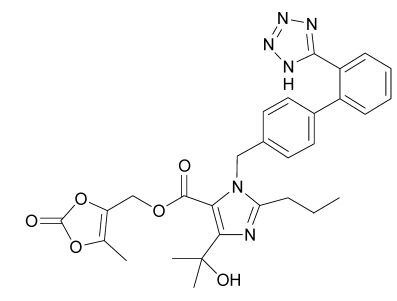

Olmesartan medoxomil (OLM)

Fig. 1. Chemical Structures of Cefmetazole, Cefpodoxime Proxetil and Olmesartan Medoxomil

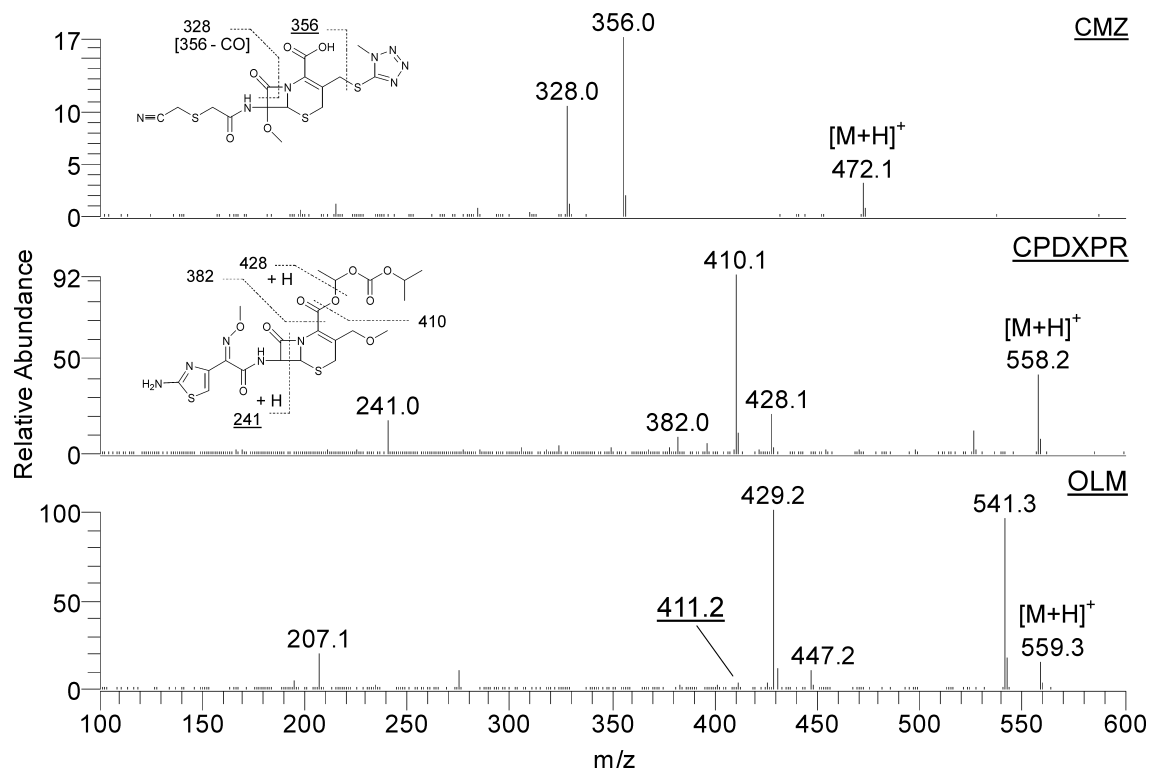

Fig. 2. MS/MS Spectra of Cefmetazole, Cefpodoxime Proxetil and Olmesartan Medoxomil 


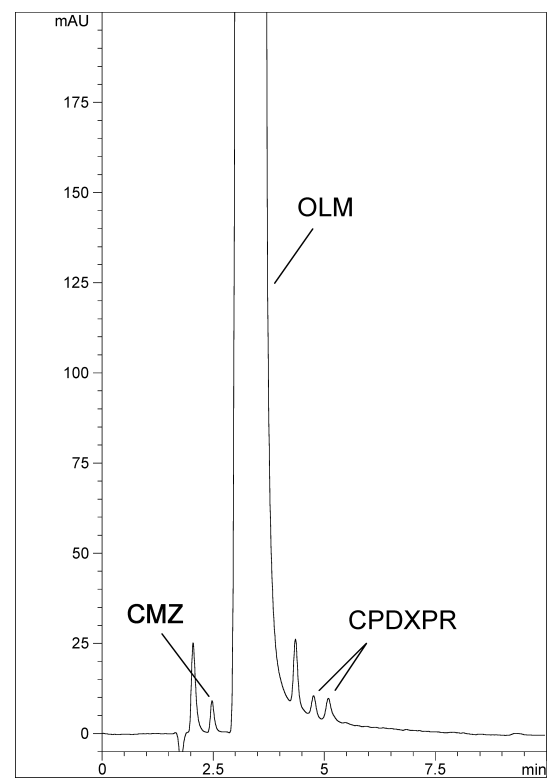

Fig. 3. Chromatogram of Olmesartan Medoxomil Spiked with $0.1 \%$ Cefmetazole and Cefpodoxime Proxetil Monitored at UV $250 \mathrm{~nm}$

selected as a monitor ion for CPDXPR with collision energy of $22 \mathrm{eV}$. In addition to that, a divert valve was connected between the analytical column and the MS, and the OLM peak was eliminated from the system. These changes enabled detection of trace amounts of CMZ and CPDXPR in OLM tablets. The chromatograms with different MS/MS conditions are shown in Fig. 4.

Validation of Analytical Method According to Human Drug CGMP Notes (Vol. 7, No. 1) by the U.S. Food and Drug Administration (FDA), the FDA requires the detection of penicillin $\mathrm{G}$ contamination at a level of $0.03 \mathrm{ppm}$ as the violative detection amount at least. Thus, validation study was conducted to evaluate the ability of the developed analytical method at around this level.

Non-contaminated OLM tablets were evaluated to verify the presence of any interference peaks from the OLM tablets for detection of CMZ or CPDXPR peaks. No interference peaks from OLM tablets were observed in the detection of the CMZ or CPDXPR peaks, therefore specificity of the method was achieved.

The detection limit was estimated from the signal-to-noise $(\mathrm{S} / \mathrm{N})$ ratio by analysis of diluted CMZ and CPDXPR solutions. According to the result, the observed detection limit $(\mathrm{S} / \mathrm{N} 3)$ of those two compounds was $0.05 \mathrm{ng} / \mathrm{ml}$ (equivalent to about $0.002 \mathrm{ppm}$ ). Based on the assumption that the sensitivities of CMZ and CPDXPR are the same as that of penicillin $G$, this sensitivity of the method would meet the requirement stated in Human Drug CGMP Notes (Vol. 7, No. 1), that the sensitivity of the analytical method should be similar to that of $0.006 \mathrm{ppm}$ for penicillin G. Therefore, sensitivity of this method was considered satisfactory for verifying the contamination of CMZ and CPDXPR to OLM tablets.

The linearity of the method was verified for the concentrations in the range of 0.325 to $3.25 \mathrm{ng} / \mathrm{ml}$ (corresponding to 0.015 to $0.15 \mathrm{ppm}$ ). The relationship between the peak area response and concentration was subjected to linear regression analysis. The corresponding linear regression equations
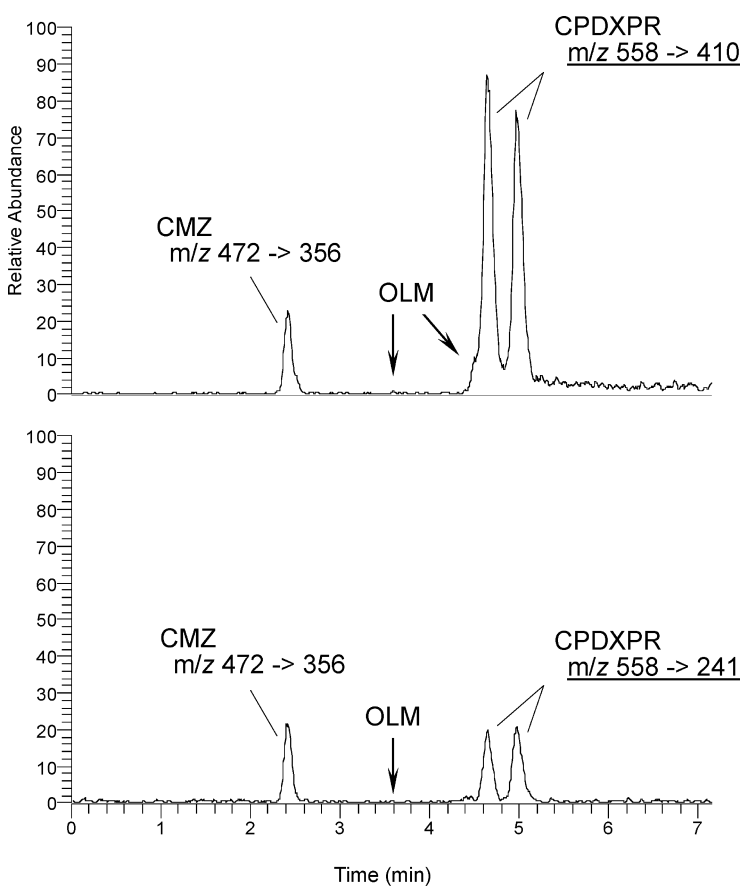

Fig. 4. Chromatogram Olmesartan Medoxomil Spiked with $0.1 \mathrm{ppm}$ Cefmetazole and Cefpodoxime Proxetil Obtained by LC-MS/MS with Different Monitoring Ions

Table 1. Accuracy and Precision Data for Cefmetazole and Cefpodoxime Proxetil Determination in Olmesartan Medoxomil Tablets

\begin{tabular}{|c|c|c|c|c|c|}
\hline & \multirow{2}{*}{$\begin{array}{c}\text { Added } \\
(\mathrm{ng})^{a)}\end{array}$} & \multicolumn{2}{|c|}{ Observed (ng) } & \multicolumn{2}{|c|}{ Recovery (\%) } \\
\hline & & Mean & S.D. & Mean & R.S.D. \\
\hline \multicolumn{6}{|l|}{$5 \mathrm{mg}$ tablet } \\
\hline $\mathrm{CMZ}$ & 3.25 & 3.28 & 0.272 & 100.1 & 8.57 \\
\hline CPDXPR & & 3.09 & 0.191 & 94.2 & 5.81 \\
\hline \multicolumn{6}{|l|}{$20 \mathrm{mg}$ tablet } \\
\hline $\mathrm{CMZ}$ & 6.50 & 6.34 & 0.560 & 96.7 & 8.69 \\
\hline CPDXPR & & 5.91 & 0.290 & 90.2 & 4.41 \\
\hline \multicolumn{6}{|l|}{$40 \mathrm{mg}$ tablet } \\
\hline $\mathrm{CMZ}$ & 13.00 & 13.39 & 0.623 & 102.2 & 4.72 \\
\hline CPDXPR & & 11.65 & 0.379 & 88.9 & 2.96 \\
\hline
\end{tabular}

Each data value is the result of six replicated determinations. a) Equivalent to violative detection amount of $0.03 \mathrm{ppm}$ penicillin $\mathrm{G}$ in corresponding tablets.

obtained were $y=1784.0 x-56.3$ with a correlation coefficient of 0.9991 for $\mathrm{CMZ}$, and $y=2568.5 x-40.4$ with a correlation coefficient of 0.9993 for CPDXPR. These results show good linear correlation between the peak area response and concentration for both compounds.

In order to demonstrate the accuracy of the method at around the violative detection amount, corresponding amounts of CMZ and CPDXPR were added to OLM tablets and their recoveries were determined. According to the results shown in Table 1, acceptable recoveries of CMZ and CPDXPR were achieved.

The precision was evaluated by six replicate determinations of CMZ and CPDXPR in spiked sample. The standard deviation of the amounts of CMZ and CPDXPR determined are also shown in Table 1 and acceptable precision was obtained for the developed method.

Consequently, the method developed is sufficiently accurate and precise for verifying CMZ and CPDXPR contamina- 

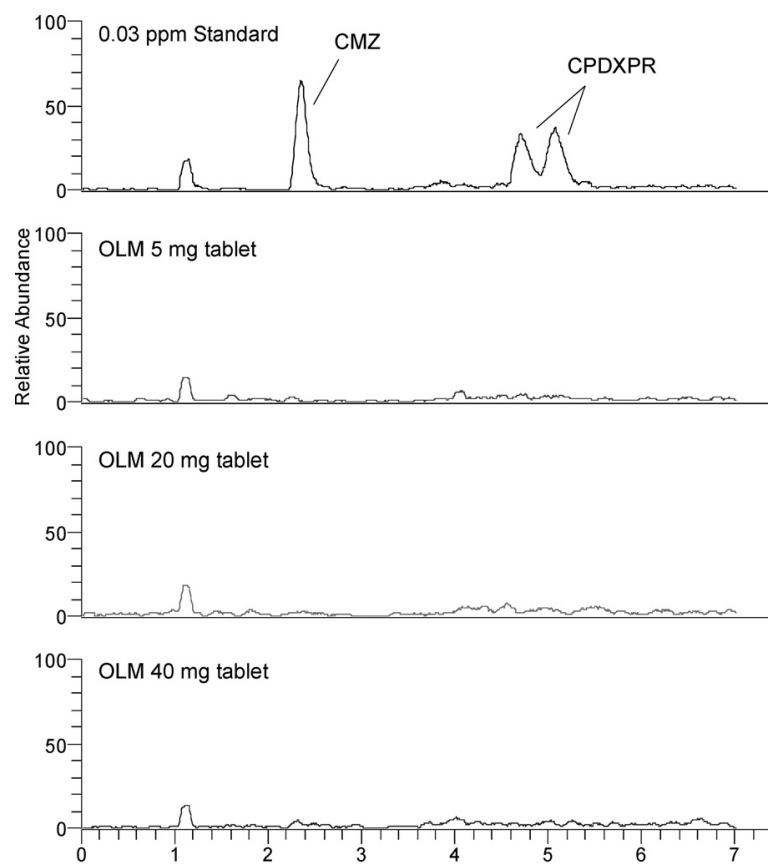

Fig. 5. Determination of Cefmetazole and Cefpodoxime Proxetil in Olmesartan Medoxomil Tablets

tion in OLM tablets at the level of regulatory requirement.

Determination of CMZ and CPDXPR Contaminants in OLM Tablets Determination of CMZ and CPDXPR contaminants in actually manufactured OLM tablets was performed with the developed method. As the chromatograms show in Fig. 5, no detectable CMZ or CPDXPR was found in the OLM tablets. Therefore, it was confirmed that no contamination of CMZ or CPDXPR to OLM tablets occurred at the facility, and, furthermore, adequacy of the cleaning procedure for the manufacturing equipment and control of manufacturing environment were verified.

\section{Conclusions}

An LC-MS/MS method for the verification of CMZ and CPDXPR contamination in OLM tablets was established. According to the results of the validation of the method, the method was found to be selective, sensitive, accurate and precise. The limit of detection of $0.002 \mathrm{ppm}$ obtained for both compounds in this method satisfies the regulatory requirement for the detection of $\beta$-lactam antibiotic contamination. Using this developed method, CMZ and CPDXPR contaminants in actually manufactured OLM tablets was investigated and no detectable CMZ or CPDXPR was found. The results indicate that no contamination occurred in the manufacturing facility, suggesting that this method would be useful for detecting contamination of non- $\beta$-lactam pharmaceuticals with CMZ and CPDXPR.

\section{References}

1) Weber W. W., Grossman M., Thom J. V., Sax J., Chan J. J., Duffy M. P., N. Engl. J. Med., 268, 411-415 (1963).

2) A Review of Procedures for the Detection of Residual Penicillins in Drugs, U.S. Food and Drug Administration, November 1977.

3) Saxon A., Beall G. N., Rohr A. S., Adelman D. C., Ann. Intern. Med. 107, 204-215 (1987).

4) Dewdney J. M., Maes L., Raynaud J. P., Blanc F., Scheid J. P., Jackson T., Lens S., Verschueren C., Food Chem. Toxicol., 29, 477-483 (1991).

5) Miles A. M., Bain B., J. Assoc. Acad. Minor. Phys., 3, 50-56 (1992).

6) Romano A., Torres M. J., Namour F., Mayorga C., Artesani M. C., Venuti A., Guéant J. L., Blanca M., Allergy, 57 (Suppl. 72) 52-57 (2002).

7) Guidance for Industry: Manufacturing, Processing, or Holding Active Pharmaceutical Ingredients, U.S. Food and Drug Administration, March 1998.

8) Guide to Inspections Validation of Cleaning Processes, U.S. Food and Drug Administration, July 1993.

9) Guide to Inspections of Bulk Pharmaceutical Chemicals, U.S. Food and Drug Administration, September 1991.

10) Mirza T., Lunn M. J., Keeley F. J., George R. C., Bodenmiller J. R., J. Pharm. Biomed. Anal., 19, 747-756 (1999).

11) Lambropoulos J., Spanos G. A., Lazaridis N. V., J. Pharm. Biomed. Anal., 23, 421-428 (2000)

12) Nozal M. J., Bernal J. L., Toribio L., Martín M. T., Diez F. J., J. Pharm. Biomed. Anal., 30, 285-291 (2002).

13) Klinkenberg R., Streel B., Ceccato A., J. Pharm. Biomed. Anal., 32, $345-352$ (2003).

14) Holmes A. J., Vanderwielen A. J., PDA J. Pharm. Sci. Technol., 51, 149-152 (1997)

15) Fukutsu N., Konse T., Kawasaki T., Saito K., Nakazawa H., J. Pharm. Biomed. Anal., 41, 599-602 (2006).

16) Fukutsu N., Sakamaki Y., Kawasaki T., Saito K., Nakazawa H., J Pharm. Biomed. Anal., 41, 1243-1250 (2006). 